\title{
Bjorken Flow of the quark-gluon plasma and Gauge/Gravity Correspondence
}

\author{
R. A. Janik \\ M.Smoluchowski Institute of Physics, Jagellonian University, \\ Reymonta 4, 30-059 Krakow, Poland \\ E-mail: ufrjaniketh.if.uj.edu.pl
}

\section{Robi Peschanski*}

Institut de Physique Théorique and URA 2306, Unité de Recherche associée au CNRS, CEA-Saclay, F-91191 Gif/Yvette Cedex, France

E-mail: robi.peschanski@cea.fr

\begin{abstract}
The contribution presents a brief summary of the Gauge/Gravity approach to the study of hydrodynamic flow of the quark-gluon plasma formed in heavy-ion collisions, in a boost-invariant setting (Bjorken flow). Considering the ideal case of a supersymmetric Yang-Mills theory for which the AdS/CFT correspondence gives a precise form of the Gauge/Gravity duality, the properties of the strongly coupled expanding plasma are put in one-to-one correspondence with the metric of a 5-dimensional black hole with the horizon moving away in the 5th dimension and its deformations consistent with the relevant Einstein equations. Several recently studied aspects of this framework are recalled and put in perspective. New results in collaboration with G. Beuf and M. Heller on the early time expansion towards the hydrodynamical regime are provided giving a new insight on the far-from-equilibrium behaviour of the fluid at strong coupling and the thermalization and isotropization problems.
\end{abstract}

European Physical Society Europhysics Conference on High Energy Physics, EPS-HEP 2009,

July $16-222009$

Krakow, Poland

\footnotetext{
*Speaker.
} 


\section{Gauge/Gravity correspondence and AdS/CFT}

The correspondence between Gauge field theories and Gravity has become a general tool for investigating problems where QCD perturbative calculations are not expected to work and strong coupling seems required. Among those problems, the hydrodynamic behaviour of the Quark-Gluon Plasma (QGP) observed in heavy-ion reactions at RHIC is one of the most prominent. Our goal is to use the Gauge/Gravity duality in this context.

Gauge/Gravity duality can be very qualitatively understood in the following intuitive way: in the framework of 10-dimensional string theory, the exchange of a graviton is equivalent to the oneloop contribution of gauge bosons, since they both are represented by the same cylindrical surface. Moreover, while the large distance (long cylinder) contribution is particularly simple in the gravity language, since it amounts to a one-graviton exchange, it is quite involved in gauge theory, since it deals with a quantum one-loop, long open-string, large-distance exchange.

However, for more quantitative study, it is necessary to give a precise meaning to this relationship and we are led to consider the paradigmatic case of the Anti de Sitter/ $\mathscr{N}=4$ Supersymmetric Yang-Mills theory duality, or in short the AdS/CFT correspondence [1]. This duality involves a conformal field theory (CFT) and thus has no typical scale, contrary to QCD, where e.g. $\Lambda_{Q C D}$ plays the role of a confinement scale. However, one may expect that lessons from the $\mathscr{N}=4$ Supersymmetric Yang-Mills theory may be relevant for the deconfined phase of a QCD plasma produced in a high-energy heavy-ion collision. One has to be careful, however, about which observables can be discussed in this way. The bulk properties of a QGP are among those where the AdS/CFT correspondence gives some new insight, as we shall see now.

\section{Late Time Dynamics}

The main practical tool that we will use is the so-called holographic renormalization [2]. It allows one to relate the properties of the 4-dimensional Minkowskian boundary representing the physical space-time to properties of gravity in the 5-dimensional AdS space (the 5 other dimensions of the string theory play a fundamental role for the consistency of the scheme but not in our calculations). One writes

$$
d s^{2}=\frac{g_{\mu v}(z) d x^{\mu} d x^{v}+d z^{2}}{z^{2}} \quad g_{\mu v}=g_{\mu v}^{(0)}\left(=\eta_{\mu v}\right)+z^{2} g_{\mu \nu}^{(2)}(=0)+z^{4}\left\langle T_{\mu v}\right\rangle+z^{6} \ldots
$$

In (2.1), $g_{\mu v}(z)$ is the Fefferman-Graham (FG) 5-dimensional metric, $\left\langle T_{\mu v}\right\rangle$ being the expectation value of the physical 4-dimensional stress-energy tensor. The holography property, that is the one-to-one correspondence between the $4 \mathrm{~d}$-boundary and the $5 \mathrm{~d}$-bulk is here realized by the property that all subsequent coefficients $z^{6} \ldots$ of the $z$-expansion in the fifth dimension, are determined by $\left\langle T_{\mu v}\right\rangle$ via the 5-dimensional Einstein equations, provided the stress-energy tensor verifies the tracelessness and energy-momentum conservation relations.

The main goal of our approach [3] is to derive the gravity duals of an expanding fluid corresponding to the plasma phase of the $\mathscr{N}=4$ Supersymmetric Yang-Mills theory in the relativistic kinematics proper to a heavy-ion reaction. In this section we recall previous results of our approach concerning the late-time properties of a boost-invariant flow. Indeed, the complexity of the Einstein 
equations to be solved is reduced by the boost-invariant symmetry and the existence of a hierarchy between the coefficients of the expansion (2.1) due to a scaling property. Starting with a family of stress-energy tensors

$$
\left\langle T_{\mu \nu}\right\rangle=\operatorname{Diag}\left\{\varepsilon(\tau), \frac{d}{d \tau} \varepsilon(\tau)-\tau^{2} \varepsilon(\tau), \varepsilon(\tau)+\frac{1}{2} \tau \frac{d}{d \tau} \varepsilon(\tau), \varepsilon(\tau)+\frac{1}{2} \tau \frac{d}{d \tau} \varepsilon(\tau)\right\},
$$

expressed in the $\tau-\eta$ proper-time-spatial rapidity frame, with $\varepsilon(\tau) \propto \tau^{-s}$ and applying the holographic renormalization by resumming Eq.(2.1), one realizes that the only consistent (i.e. nonsingular) solution is given by $s \equiv \frac{4}{3}$, which corresponds to the perfect fluid (or Bjorken flow). Moreover for that value, the obtained FG metric is a Black Hole one (more precisely a black brane) with the horizon moving away in the $z$-direction, in a holographic correspondence with the falling temperature due to cooling of the plasma. Indeed, precise duality relations exist at large $\tau$ between the moving Black Hole and the cooling Plasma, namely

$$
\text { Horizon }: z_{h} \propto \tau^{\frac{1}{3}} \quad \text { Temperature }: \sim \tau^{-\frac{1}{3}} \quad \text { Entropy } \sim \text { Area }: \sim \tau \cdot 1 / z_{h}^{3}=\text { const } .
$$

One recognizes here the properties of an ideal Bjorken flow.

Taking into account the asymptotic limit of the perfect fluid, it has been possible to derive an expansion at large $\tau$, allowing to derive the dissipative corrections of the flow due to dissipation and transport through the dual gravitational properties. The main ingredients are the same, i.e. the expansion of Einstein equations and the consistency of the holographic renormalization requiring nonsingular geometries. For instance, beyond the perfect fluid, one can compute the shear viscosity through the equation $\partial_{\tau} \varepsilon=-\frac{4}{3} \frac{\varepsilon}{\tau}+\frac{\eta}{\tau^{2}}+\cdots \Rightarrow \frac{\eta}{s}=\frac{1}{4 \pi}$, which gives the same result as the classical static calculations.

\section{Early Time Dynamics}

The problem of early-time dynamics, even with the boost-invariance symmetry assumption, appears to be more involved. In fact one is faced with the full set of nonlinear Einstein equations

$$
R_{A B}-\frac{1}{2} R \cdot G_{A B}-6 G_{A B}=0
$$

where $R_{A B}$ is the Ricci tensor and the FG metric $G_{A B}$ is given by

$$
d s^{2}=G_{A B} d x_{A} d x_{B}=\frac{-e^{a(\tau, z)} d \tau^{2}+\tau^{2} e^{b(\tau, z)} d y^{2}+e^{c(\tau, z)} d x_{\perp}^{2}}{z^{2}}+\frac{d z^{2}}{z^{2}}
$$

Indeed, one is physically dealing with a non-(even far-from) equilibrium fluid whose theoretical properties and formalism are hardly known. It is a remarkable but challenging feature of Gauge/Gravity duality to give first hints on this difficult problem. In a very recent work [4], we have obtained some results following the same path as for late time (holography and nonsingularity of the geometry) but with quite different features from the late-time solution.

- No scaling at early time. In fact, there appears to be no hierarchy at small $\tau$ between the coefficients of the holographic expansion (2.1). The full content of the Einstein equations has to be taken into account. 

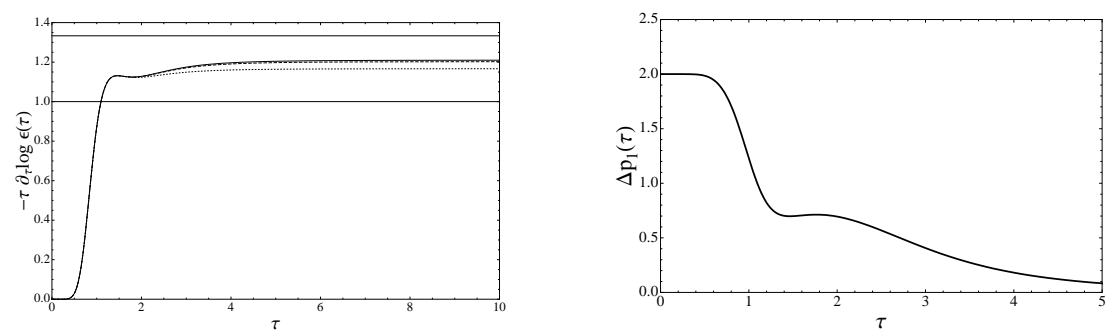

Figure 1: Left: The index $s$ from Energy density; Right: Pressure anisotropy.

- Dependence on initial conditions. Due to the absence of scaling, there is no unique geometry analogous to the moving Black Hole solution of late time. In physical terms, the far-from equilibrium pre-asymptotic regime of the flow is mainly governed by the initial conditions, and in the dual set-up by the gravitational response to them.

- The metric is singular at all times. Quite unexpectedly, it is possible to derive a general mathematical consequence of the Einstein equations (essentially from the $G_{z z}$ component) following which there should be a singularity in $z$ at all proper-time $\tau$ in the metric (3.2). Hence some kind of non-trivial horizon exists from the beginning of the flow, which can be interpreted as the "ancestor" of the BH horizon of the late-time solution.

- The geometry should stay regular. Following the same consistency requirement as previously, one is led to impose the constraint of a regular geometry, meaning that the singularity in the metric is only a coordinate singularity with the curvature scalars (such as $\mathfrak{R}^{2}$ ) remaining bounded analogously as for a Schwarzschild horizon.

In practice, one method is to solve the Einstein equations (3.1) in a power series with an appropriate ansatz for the initial conditions e.g. for the coefficient $a(z, \tau=0)$ of the metric (3.2). Among the first obtained results, we show in fig. 1, the result for the index $s=\frac{d \log \varepsilon(\tau)}{d \tau}$ and the pressure anisotropy $\Delta p(\tau)=1-\frac{p_{\|}(\tau)}{p_{\perp}(\tau)}$ for one typical solution. A quite general feature seems to emerge consisting in a quite fast evolution to thermalization at the beginning but with eventually a partial isotropization, as shown in fig. 1. However, since it was necessary to perform a resummation procedure, before a firm conclusion is reached, a complete study using direct numerical solution of Einstein's equations has to be made.

Acknowledgements: RJ was supported by Polish science funds during 2009-2011 as a research project (NN202 105136).

\section{References}

[1] J. M. Maldacena, Adv. Theor. Math. Phys. 2, 231 (1998)

[2] K. Skenderis, Class. Quant. Grav. 19, 5849 (2002).

[3] R. A. Janik and R. B. Peschanski, Phys. Rev. D 73, 045013 (2006).

[4] G. Beuf, M. P. Heller, R. A. Janik and R. Peschanski, arXiv:0906.4423 [hep-th]

"Boost-invariant early time dynamics from AdS/CFT", to appear in JHEP. 